Advancements in Instrumentation

\title{
Development of an Electrochemical Cholesterol Sensor System for Food Analysis
}

\author{
Tsutomu Nagaoka, ${ }^{* \dagger}$ Shiho Tokonami, ${ }^{*}$ Hiroshi Shitgi,* Hiroaki Matsumoto,* \\ Yasuhiro TAKAGI, $* * *$ and Yasunori TAKAHASHI $* * *$ \\ *Department of Applied Chemistry, Graduate School of Engineering, Osaka Prefecture University, \\ 1-2 Gakuen-cho, Naka, Sakai 599-8570, Japan \\ **Nanoscience and Nanotechnology Research Center, Research Organization for the 21th Century, \\ Osaka Prefecture University, 1-2 Gakuen-cho, Naka, Sakai 599-8570, Japan \\ ***JSK Co. Ltd., 1-5-1 Higashi-amakawa, Takatsuki, Osaka 560-0012, Japan
}

\begin{abstract}
In this article, we report on a food-cholesterol monitoring sensor based on a non-enzymatic approach. Amorphous and single-crystal gold electrodes were modified with an alkanethiol self-assembled monolayer to quantify it by voltammetry. We first discuss the basic characteristics of these sensors and provide more information about the instrument developed by JSK Co. This instrument is a battery-operated handheld voltammetric analyzer, which mounts a sensor chip to monitor cholesterol contents in food samples. The sensor showed excellent linearity with the cholesterol concentration; egg-yolk samples were analyzed to give an excellent agreement between the values obtained by the sensor $(1.4 \mathrm{mM})$ and chromatography $(1.1 \mathrm{mM})$.
\end{abstract}

(Received October 7, 2011; Accepted December 19, 2011; Published February 10, 2012)

\section{Introduction}

Currently, there is a surge in demand for the real-time monitoring of chemical compounds, which is required for various types of critical operations, such as detecting food poisoning and chemical/biological threat agents. Many chemical sensors have been developed for these purposes as well as for avoiding labor-intensive analytical operations. However, developing a sensor device is made very difficult by the requirement that, based on its selectivity scheme, the device must discriminate one particular molecule or certain types of molecules of interest out of the large number of other molecules existing in a sample. Due to this difficulty, highly selective sensors are mostly fabricated using biologically acquired ligands, such as enzymes and antibodies. ${ }^{1-7}$ However, these ligands are usually expensive and prone to lose their activities for prolonged use. It is therefore desired to make use of artificial receptors possible, especially in low-cost sensing applications as well as in harsh chemical and/or physical environments. ${ }^{8}$ However, such receptors often have serious problems with insufficient selectivities against target molecules.

To circumvent this dilemma, we have recently developed sensing devices based on molecularly imprinted polymers using overoxidized conducting polymers, which can discriminate amino acid enantiomers, structural isomers, etc. in highly efficient manners. ${ }^{9-14}$ We have also developed self-assembled monolayer (SAM) based sensors for the detection of vitamin K1 and skin cholesterol. ${ }^{15-17}$

Cholesterol is essential for many metabolic processes, but

$\dagger$ To whom correspondence should be addressed.

E-mail: nagaoka@chem.osakafu-u.ac.jp high levels are often associated with heart disease. To know of its level in food as well as in our body, a number of papers related to electrochemical cholesterol sensors have been published based on enzymes, ${ }^{18-22}$ molecularly imprinted polymers, ${ }^{16,17,23,24}$ and other non-enzymatic approaches..$^{25,26}$ This article presents a SAM-based sensor optimized for food cholesterol. The monolayer, structured on a gold substrate, has high selectivity for cholesterol to provide a convenient means for developing cost-effective sensors especially suited for disposable use. Based on these results examined in this study, JSK Co. has recently marketed a handheld sensor system for food cholesterol analysis; the performance of this system is discussed in this article.

\section{Experimental}

\section{Chemicals and apparatus}

Cholesterol and its derivatives were obtained from Wako Chemicals, Sigma, and Tokyo Chemical Industry. Other chemicals were of the reagent grade and used without further purification. Milli-Q grade water $\left(>18.3 \mathrm{M} \Omega \mathrm{cm}^{-1}\right)$ was used throughout. Voltammetric measurements were conducted with either a Hokuto Denko Model HZ03000 potentiostat or an ALS Model 842 analyzer at ambient temperature. The surface of the electrode was imaged with a Nanoscope III scanning microscope (Veeco). The potential was referred to a $\mathrm{Ag}|\mathrm{AgCl}|$ sat. $\mathrm{KCl} \|$ electrode throughout.

\section{Preparation of self-assembly electrode}

We prepared the SAM on amorphous and (1 111 ) single-crystal gold using $\mathrm{C}_{18} \mathrm{H}_{37} \mathrm{SH}$ and cholesterol. The thiol, $\mathrm{C}_{18} \mathrm{H}_{37} \mathrm{SH}$, is hereafter abbreviated as $\mathrm{C} 18 \mathrm{SH}$. For the amorphous gold 
modification, a gold disk electrode $(1.6 \mathrm{~mm}$ in diameter, purchased from BAS Co.) was mechanically and electrochemically polished before the modification. Afterwards, the electrode was rinsed with water, followed by immersion in an ethanol solution containing $20 \mathrm{mM}$ cholesterol and $0.10 \mathrm{mM}$ $\mathrm{C} 18 \mathrm{SH}$ for $30 \mathrm{~min}$ to structure the thiol-cholesterol intermixed SAM on the gold (see Fig. 1A). The electrode was then immersed in stirred ethanol for $30 \mathrm{~min}$ to wash cholesterol out of the SAM. Detailed experimental procedures have been given elsewhere. ${ }^{16}$ A microelectrode (BAS, $10 \mu \mathrm{M}$ in diameter) was similarly treated and used in the same way for a sensitivity comparison. To compare selectivity, we also prepared the SAM on a $1 \times 10^{-2} \mathrm{~cm}^{2}$ Auro Sheet (1 111$) \mathrm{FE}$ (supplied from Tanaka Kikinzoku Kougyou; the coverage of (1 111$)$ surface area over the total area is nominally $>95 \%$ ). This sheet was treated in the same way as mentioned above, but used without mechanical polishing.

For investigating the cholesterol response, the washed electrode, possessing slots with a size compatible to cholesterol, was immersed in an ethanolic solution of cholesterol for $30 \mathrm{~s}$ and rinsed with an ample amount of water. The cholesterol concentration was $1.0 \mathrm{mM}$, unless otherwise noted. The electrode rebinding the cholesterol was then subjected to voltammetric analysis in an aqueous $50 \mathrm{mM} \mathrm{KClO}_{4}$ solution containing $5.0 \mathrm{mM} \mathrm{K} \mathrm{K}_{4} \mathrm{Fe}\left(\mathrm{CN}_{6}\right)$, which was added as a redox marker. To study the responses from interfering molecules, the electrode was soaked in a solution containing the interfering molecule of $1.0 \mathrm{mM}$ for $30 \mathrm{~s}$, and the same voltammetric procedures were conducted.

\section{Cholesterol determination using the developed instrument,} CholestWatcher

The quantification procedure by this instrument consists of two steps; measurements of the base current and the sample cholesterol current. The first step actually monitors the current from the discharge of the marker, reaching through the slots in the SAM that are unoccupied with cholesterol. The second step monitors the discharge current, which is decreased by the rebound cholesterol that blocks the slots. The detailed procedures are as follows: Firstly, a chip electrode fabricated on a glass strip is initialized by submerging it into ethanol for $5 \mathrm{~min}$. As in the above first step, the instrument measures the voltammetric current after applying a $100-\mu \mathrm{L}$ solution kit A, which contains the marker and base electrolyte, on the chip. As the second step, the electrode chip is soaked into a cholesterol sample to take cholesterol up into the monolayer, and then the chip surface is washed with water. The current is measured after applying a $100-\mu \mathrm{L}$ solution A onto the chip. Each data acquisition takes $100 \mathrm{~s}$, and the instrument outputs the cholesterol concentration to its LCD screen. The obtained value is stored in the instrument memory for later use.

\section{Egg yolk analysis}

Egg yolk was manually separated from albumen and its $0.5 \mathrm{~g}$ portion was dissolved in $0.5 \mathrm{~mL}$ ethyl acetate and vortexed for $10 \mathrm{~min}$. The mixture was centrifuged for $5 \mathrm{~min}$ at $6500 \mathrm{rpm}$, and the upper layer was pipetted off and diluted with 19 parts of ethanol. The thus-prepared egg yolk solution was subjected to voltammetric analysis by the procedures described above, and the quantized value was compared to that obtained by gas chromatographic analysis, which was conducted by Japan Food Research Laboratories.
(A)
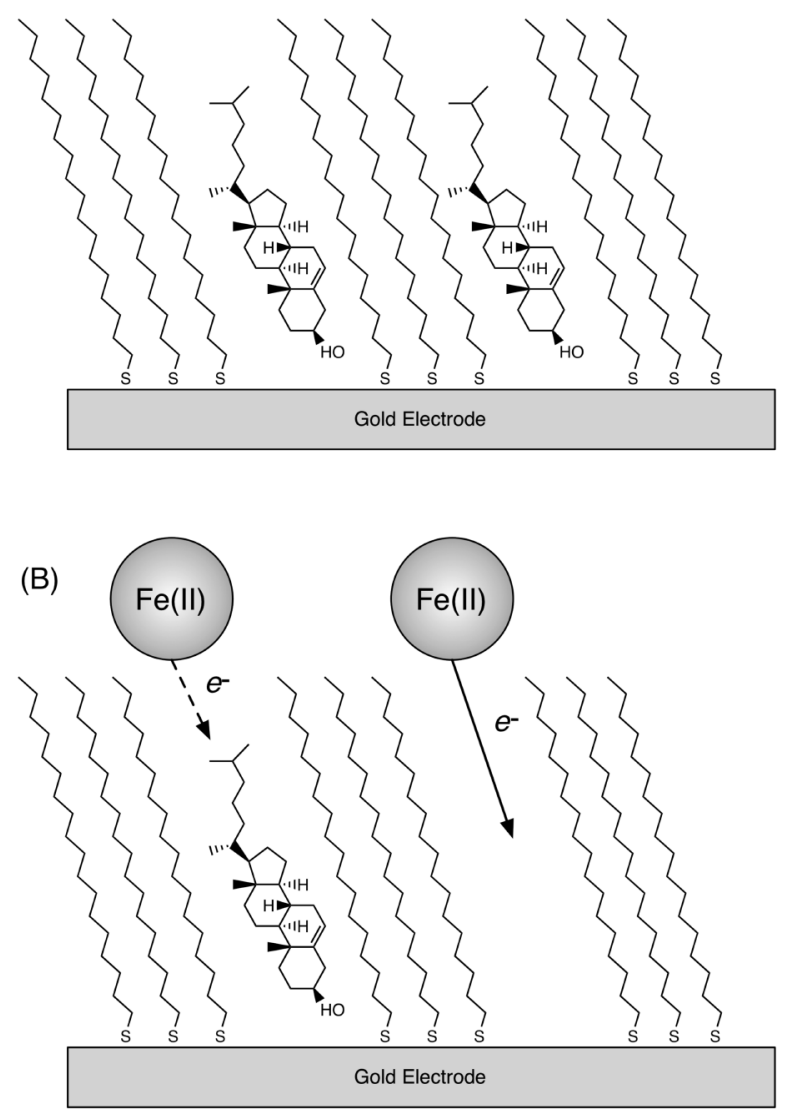

Fig. 1 SAM fabricated for the detection of cholesterol. (A) Formation of the SAM intermixed with $\mathrm{C} 18 \mathrm{SH}$ and cholesterol on a gold substrate, and (B) detection of cholesterol by discharge of the redox marker complex ion, $\mathrm{Fe}(\mathrm{II})$.

\section{Results and Discussion}

\section{Basic characteristics of the thiol modified electrodes}

Figure 1 shows an illustrative image of the electrode surface modified with $\mathrm{C} 18 \mathrm{SH}$. The gold surface self-assembled with a mixture of $\mathrm{C} 18 \mathrm{SH}$ and cholesterol (A) creates a totally insulated and compact monolayer, as shown in the voltammetric response of curve b in Fig. 2. The cholesterol-C18SH intermixed SAM layer almost completely lost the marker signal $30 \mathrm{~min}$ after the immersion of the electrode in the ethanolic $100 \mu \mathrm{M} \mathrm{C} 18 \mathrm{SH}+20$ $\mathrm{mM}$ cholesterol solution, suggesting that the surface was saturated with these molecules. Washing out the cholesterol with ethanol leaves hydrophobic slots created by the template cholesterol molecule. ${ }^{16,17,23}$ Surface characterization imaged by AFM is given in Fig. S1 in Supporting Information.

Although the response of the washed electrode was not fully recovered, the electrode showed a distinctive response (curves a and $\mathrm{c}$ in Fig. 2). It should be noted here that cholesterol requires a proper redox marker to be detected by electrochemical techniques, unless a highly positive potential is applied. Rebinding of cholesterol clogs some of the slots, the number of which is dependent on the cholesterol concentration in a sample solution, and prevents access of the marker (or electrons from it) to the electrode surface. The quantification of cholesterol by this scheme basically depends on the number of the blocked slots, which explains the negative dependence of the response on the cholesterol concentration. ${ }^{16}$ Table 1 summarizes the 


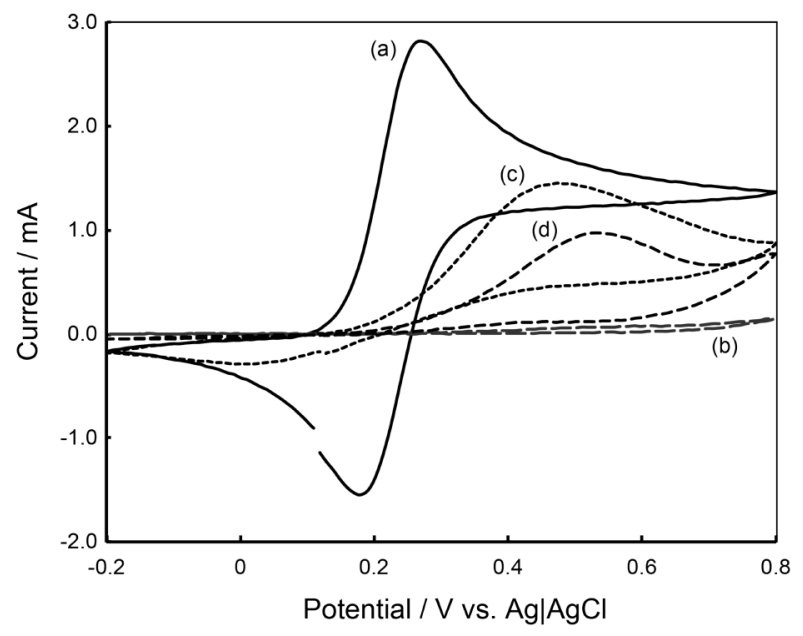

Fig. 2 Cyclic voltammetric responses of the $5.0 \mathrm{mM} \mathrm{Fe}(\mathrm{CN})_{6}{ }^{4}$ marker ion in $50 \mathrm{mM} \mathrm{KClO}_{4}$ on (a) unmodified electrode, (b) electrode covered with the $\mathrm{C} 18 \mathrm{SH}+$ cholesterol intermixed SAM, (c) electrode $\mathrm{b}$ after washing, and (d) electrode $\mathrm{c}$ after rebinding $1 \mathrm{mM}$ cholesterol.

Table 1 Sensitivities of amorphous and single-crystal gold electrodes for the detection of $1.0 \mathrm{mM}$ cholesterol

\begin{tabular}{lcc}
\hline \multicolumn{1}{c}{ Electrode } & $\Delta I / \mu \mathrm{A}^{\mathrm{a}}$ & $\Delta \mathrm{I}_{\mathrm{d}} / \mu \mathrm{A} \mathrm{cm}^{-2 \mathrm{~b}}$ \\
\hline Amorphous, $1.6 \mathrm{~mm}$ & 2.2 & 109 \\
Amorphous, $10 \mu \mathrm{m}$ & $0.98 \times 10^{-3}$ & 1248 \\
Single-crystal, $(111)$ & 0.92 & 92 \\
\hline
\end{tabular}

a. $\Delta I$ is defined as $\Delta I=I_{0}-I_{1}$, where $I_{0}$ and $I_{1}$ are the oxidation peak currents of the marker before and after the addition of cholesterol, respectively, and equal the peak currents for the curves $\mathrm{c}$ and $\mathrm{d}$ shown in Fig. 2.

b. $\Delta I$ divided by the electrode area.

responses of the amorphous and single-crystal electrodes. The amorphous and single-crystal electrodes showed a similar sensitivity as far as the current density is concerned, but the microelectrode gave a much higher density based on a diffusion mechanism different from that for the other two electrodes.

The voltammetric responses of the marker became less reversible upon the uptake of cholesterol in the SAM, and the cathodic peaks were notably smaller than the anodic counterparts in magnitude. Electron transfer through a tunneling barrier, such as a SAM, at distance $x$ from the electrode surface is expressed by the following equation: ${ }^{27}$

$$
k^{\circ}(x)=k^{\circ}(0) \exp (-\beta x)
$$

where $k^{\circ}(x)$, and $k^{\circ}(0)$ are the standard electron-transfer rate constants at position $x$ and at the electrode surface, and $\beta$ is a constant. Accordingly, the quasi-reversible nature would be ascribed to the reason that the marker, which cannot enter the SAM due to its bulky molecular structure, makes an electron to travel long through the pinhole (see the right-hand side marker in Fig. 1B). The slow electron transfer might also result from a discharge of the marker in the middle of the slot, if the SAM would be sufficiently flexible. The asymmetry in the peak amplitude is also notable, but it is not very clear at this stage why the decreases in the cathodic peak resulted.

Figure 3 shows the relative responses of the sensor as a function of the molecular volumes of rebound molecules. The

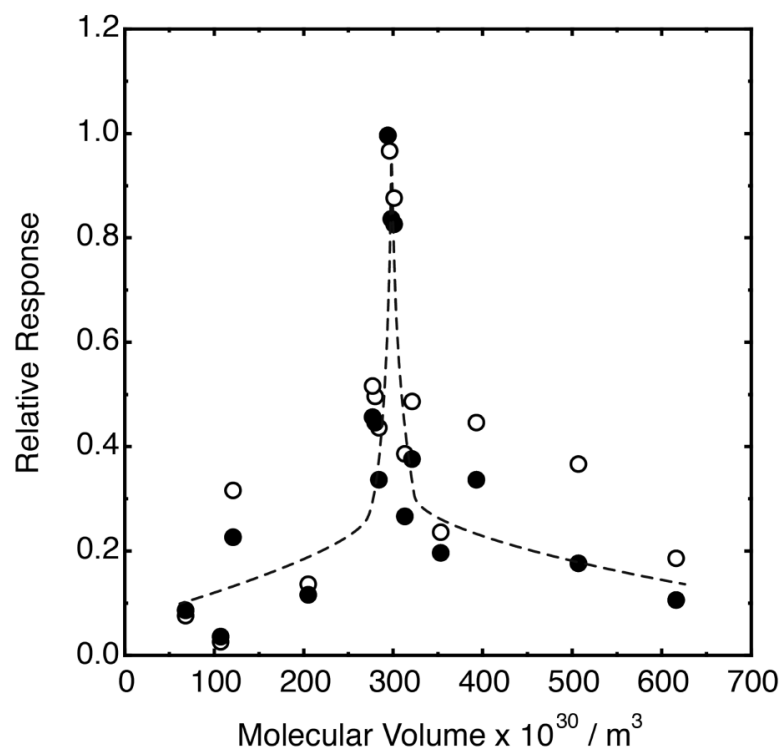

Fig. 3 Selectivity of SAM electrodes as a function of the molecular volume on the $(\bigcirc)$ amorphous and $(\bullet)$ single-crystal electrodes. The responses were normalized to the $\Delta I$ value of cholesterol for each electrode. The data plotted for the amorphous electrode were taken from a previous report. ${ }^{17}$

responses are close to unity only when the molecular volume is very close to that for cholesterol $\left(292 \times 10^{-30} \mathrm{~m}^{3}\right)$, demonstrating the high selectivity of the slot for rebound molecules; the relative responses used for plotting this figure are complied in Table S1 of Supporting Information. The excellent selectivity would be due to both the nonpolar interior wall structure of the slots and to the size of the slots created by the cholesterol template. Figure 3 also compares the selectivities of the monolayers fabricated on the amorphous and single-crystal (111) gold electrodes. ${ }^{17}$ As can be seen in the figure, better selectivities were observed for the (111) surfaces, reflecting their better-defined structures. We also compared the responses for cholesterol by varying the length of the alkyl chain from $\mathrm{C} 11 \mathrm{SH}$ to $\mathrm{C} 22 \mathrm{SH}$, and by introducing chemical groups, such as $-\mathrm{NH}_{2}$ and $-\mathrm{COOH}$, to the alkyl chains; however, the unmodified $\mathrm{C} 18 \mathrm{SH}$ gave the best result for the cholesterol selectivity. The calibration plot showed excellent linearity for the cholesterol concentration in the range of $0.2-1.0 \mathrm{mM}(R=0.997$ for the single-crystal gold, $R=0.926$ for the amorphous gold).

\section{Instrumentation}

Based on the above results, JSK Co. developed a handheld instrument and sensor chip, and marketed this system, named CholestWatcher in January 2011. Figure 4 shows photographic images of this instrument and sensor chip. The instrument is capable of mounting one sensor chip with mobile size dimensions of $50 \times 90 \times 18 \mathrm{~mm}$, is driven by a single CR2032 battery, only weighs $67 \mathrm{~g}$, can store 200 measured results in the device memory and can operate in a stand-alone mode during measurements. When connected to a host PC, the instrument is recognized as a USB peripheral device by the PC, enabling the acquired data to be transferred via a USB interface. The instrument is equipped with a 3-digit LCD panel and has three mechanical keys to execute all of the functions required for the instrument operations, such as triggering the data acquisition and manipulating the recoded data. The sensor chip $(15 \times 45 \times 0.7 \mathrm{~mm}, \quad 1.3 \mathrm{~g})$ prints 4 gold electrode pads 


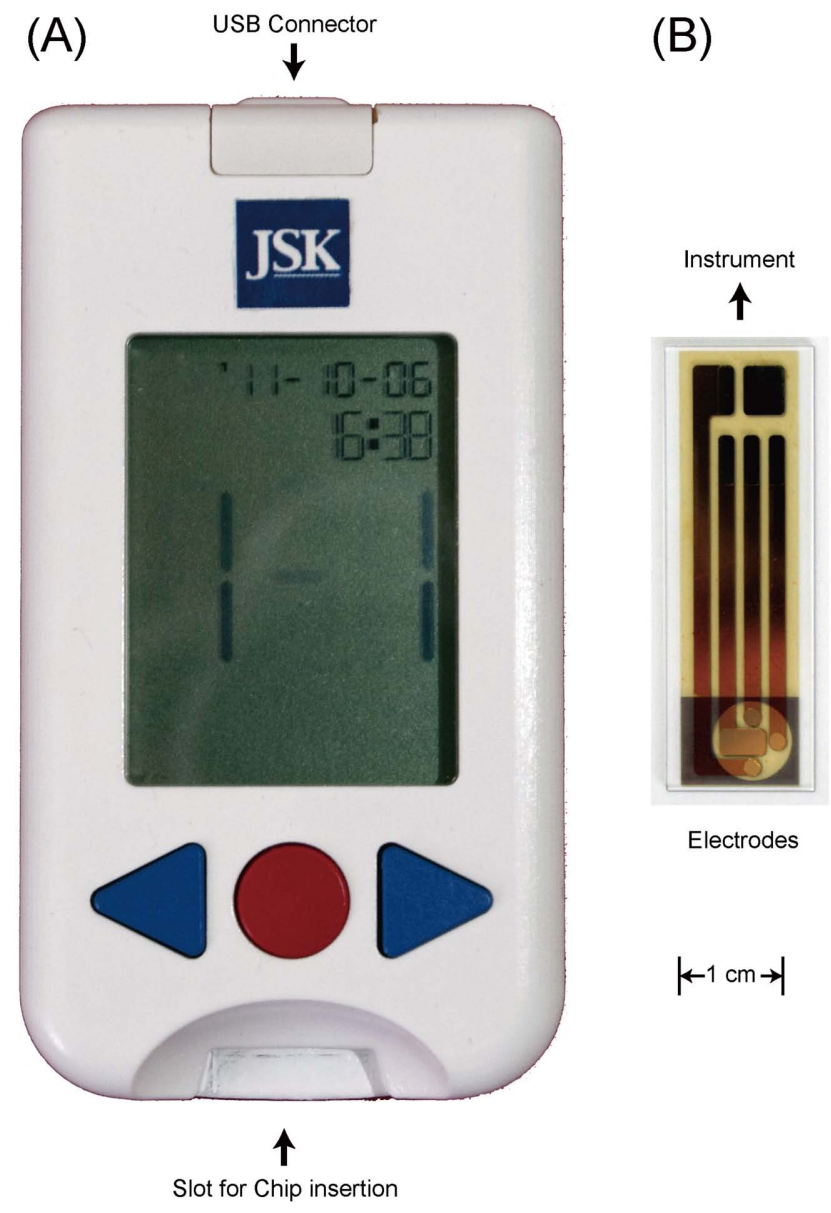

Fig. 4 Photographic images of (A) the instrument and (B) sensor chip.

(2 working electrodes, and 1 quasi-reference and counter electrodes) on a glass strip, which is highly polished until it reaches a surface roughness of $10 \mathrm{~nm}$, considering the higher selectivities observed for the single-crystal surface. Vapor deposition on the glass strip results in the formation of amorphous gold electrodes; the pads are then coated with a plastic film to expose the minimum area of the electrodes. The instrument has also been designed to work as a versatile voltammetric analyzer by inserting an unmodified electrode chip.

To determine total cholesterol in food samples, many techniques, including spectrophotometry and amperometry based on enzymatic reactions, and gas and liquid chromatographic techniques, have been reported..$^{19,28-37}$ Cholesterol in a yolk mostly exists as free cholesterol, although the exact ratio of free cholesterol to total cholesterol seems to depend on the pretreatment procedure of a yolk sample and the quantification technique employed. . $^{19,30,35}$ Although the responses of cholesterol esters showed $18-49 \%$ as high as the response of free cholesterol (see Table S1, Supporting Information), the influence of the esters on the quantized value could not be important due to the small fraction of the esters in the yolk samples. The total cholesterol concentration of $1.4 \mathrm{mM}$ for an egg-yolk sample determined by this system agreed well with the value of $1.1 \mathrm{mM}$ obtained by gas chromatography.

In conclusion, we have succeeded in developing a sensor instrument, along with a robustly designed bio-free disposable sensor chip, to provide an excellent analytical solution, which can be an alternative to more labor-intensive analytical techniques, such as chromatography, for acquiring the food cholesterol concentration.

\section{Supporting Information}

This material is available free of charge on the Web at http:// www.jsac.or.jp/analsci/.

\section{References}

1. R. O’Kennedy, W. J. J. Finlay, P. Leonard, S. Hearty, J. Brennan, S. Stapleton, S. Townsend, A. Darmaninsheehan, A. Baxter, and C. Jones, in "Sensors for Chemical and Biological Applications", ed. M. K. Ram and V. R. Bhethanabotla, 2010, Chap. 7, CRC Press, Boca Raton, 195.

2. A. Chaubey and B. D. Malhotra, Biosens. Bioelectron., 2002, 17, 441 .

3. M. Mehrvar and M. Abdi, Anal. Sci., 2004, 20, 1113.

4. Y. Wang and Y. Hasebe, Anal. Sci., 2011, 27, 401.

5. M. Liu, Y. Wen, J. Xu, H. He, D. Li, R. Yue, and G. Liu, Anal. Sci., 2011, 27, 477.

6. H. Takaoka and M. Yasuzawa, Anal. Sci., 2010, 26, 551.

7. M. T. Hossain, T. Shibata, T. Kabashima, and M. Kai, Anal. Sci., 2010, 26, 645.

8. S. Tokonami, H. Shiigi, and T. Nagaoka, Anal. Chim. Acta, 2009, 641, 7.

9. B. Deore, Z. Chen, and T. Nagaoka, Anal. Sci., 1999, 15, 827.

10. Z. Chen, Y. Takei, B. A. Deore, and T. Nagaoka, Analyst, 2000, 125, 2249.

11. B. Deore, Z. Chen, and T. Nagaoka, Anal. Chem., 2000, 72, 3989.

12. H. Okuno, T. Kitano, H. Yakabe, M. Kishimoto, B. A. Deore, H. Shiigi, and T. Nagaoka, Anal. Chem., 2002, 74, 4184.

13. H. Shiigi, H. Yakabe, M. Kishimoto, D. Kijima, Y. Zhang, U. Sree, B. A. Deore, and T. Nagaoka, Microchim. Acta, 2003, 143, 155.

14. S. Takeda, H. Yagi, S. Mizuguchi, H. Funahashi, H. Shiigi, and T. Nagaoka, J. Flow Injection Anal., 2008, 25, 77.

15. Z. Chen and T. Nagaoka, Bunseki Kagaku, 2000, 49, 543.

16. H. Shiigi, H. Matsumoto, I. Ota, and T. Nagaoka, J. Flow Injection Anal., 2008, 25, 81.

17. H. Shiigi and T. Nagaoka, Trans. Jpn. Soc. Med. Biol. Eng., 2004, 42, 181.

18. K. Vengatajalabathy Gobi and F. Mizutani, Sens. Actuators, $B, \mathbf{2 0 0 1}, 80,272$.

19. N. Peña, G. Ruiz, A. J. Reviejo, and J. M. Pingarrón, Anal. Chem., 2001, 73, 1190.

20. S. Singh, P. R. Solanki, M. K. Pandey, and B. D. Malhotra, Sens. Actuators, B, 2006, 115, 534.

21. X. Tan, M. Li, P. Cai, L. Luo, and X. Zou, Anal. Biochem., 2005, 337, 111.

22. C. Fang, J. He, and Z. Chen, Sens. Actuators, B, 2011, 155, 545.

23. S. A. Piletsky, E. V. Piletskaya, T. A. Sergeyeva, T. L. Panasyuk, and A. V. El'Skaya, Sens. Actuators, B, 1999, 60, 216.

24. A. Aghaei, M. R. Milani Hosseini, and M. Najafi, Electrochim. Acta, 2010, 55, 1503.

25. Y. Li, H. Bai, Q. Liu, J. Bao, M. Han, and Z. Dai, Biosens. Bioelectron., 2010, 25, 2356. 
26. Y. J. Lee and J. Y. Park, Biosens. Bioelectron., 2010, 26, 1353.

27. A. J. Bard and L. R. Faulkner, "Electrochemical Methods: Fundamentals and Applications", 2nd ed., 2001, Wiley, Hoboken, 624.

28. G. Pineiro-Avila, A. Salvador, and M. de la Guardia, Analyst, 1998, 123, 999.

29. C. S. Shen, I. S. Chen, and A. J. Sheppard, J. Assoc. Off. Anal. Chem., 1982, 65, 1222.

30. G. Pasin, G. M. Smith, and M. O’Mahony, Food Chem., 1998, 61, 255.

31. A. Daneshfar, T. Khezeli, and H. J. Lotfi, J. Chromatogr.,
$B, 2009,877,456$.

32. B. S. Hwang, J. T. Wang, and Y. M. Choong, J. Food Compos. Anal., 2003, 16, 169.

33. X. H. Xu, R. K. Li, J. Chen, P. Chen, X. Y. Ling, and P. F. Rao, J. Chromatogr., B, 2002, 768, 369.

34. G. C. Nogueira and N. Bragagnolo, Food Chem., 2002, 79, 267.

35. R. Z. Zhang, L. Li, S. T. Liu, R. M. Chen, and P. F. Rao, J. Food Biochem., 1999, 23, 351.

36. D. V. Maurice, S. F. Lightsey, K. T. Hsu, T. G. Gaylord, and R. V. Reddy, Food Chem., 1994, 50, 367.

37. M. Fenton and J. S. Sim, J. Chromatogr., 1991, 540, 323. 